President/Président

David Smith

University of Saskatchewan

Past President/Présidente sortante Sylvia Bashevkin

University of Toronto

President Elect/Président élu

Peter Aucoin

Dalhousie University

Secretary-Treasurer/Secrétaire-trésorier Gary O'Brien

Senate of Canada/Sénat du Canada CPSA/ACSP Secretariat \#205-1 Stewart

Ottawa, Ontario K1N 6H7

Representative of Members-at-Large/

Représentant des conseillers(ères)

John Crossley

University of Prince Edward Island

Members-at-large/Conseillers(ères)

2-year term/mandat de deux ans:

R. K. Carty (British Columbia), James J. Ciuy (University College of Cape Breton), Diane Lamoureux (Laval), Linda Trimble (Alberta), Robert Young (Western Ontario)

l-yearterm/mandat d'un an :

Keith Archer (Calgary), John Crossley (Prince Edward Island), Henry J. Jacek (McMaster), Chantal Maillé (Concordia), Katherine Teghtsoonian (Victoria)

Nominating Committee/Comité des candidatures 1995

David Smith (Saskatchewan), Chair/

Président, Maureen Covell (Simon Fraser), Réjean Landry (Laval), Frank Harrison

(St. Francis Xavier)
Invited guests/Invités

Richard Vernon (Western Ontario), Co-editor of the Journal

Guy Laforest (Laval), codirecteur de la Revue

John McMenemy (Wilfrid Laurier), Administrative Editor of the Journal/directeur administratif de la Revue

Chantal Maillé (Concordia), Chairperson of the Programme Committee/Présidente du Comité d'organisation du programme (1995)

François Houle (Ottawa), Director/ Directeur, Parliamentary Internship Programme/Programme de stage parlementaire

Clinton Archibald (Ottawa), Assistant Director/Directeur-adjoint, Parliamentary Internship Programme/Programme de stage parlementaire

Robert J. Williams (Waterloo), Ontario Legislature Intemship Programme/ Programme de stage à l'Assemblée legislative de l'Ontario

Frederick J. Fletcher (York), Chairperson, The Trust Fund/Président, Le fonds de prévoyance

Gordon Mace (Laval), Editor/Directeur du Bulletin

Manon Tremblay (Ottawa), Délégué de la Société québécoise de science politique

Chantal Maillé (Concordia), Representative to SSFC/Representante à la FCSS

Michelle Hopkins, Executive Secretary/ Secrétaire administrative

\title{
Société québécoise de science politique
}

Président, Gérard Boismenu (Montréal)

Président sortant, Jean Crête (Laval)

Vice-président, John Trent (Ottawa)

Secrétaire

Janine Krieber

5950, rue Hutchinson

Montréal, P.Q. H2V 4C1

Trésorier

Pierre P. Tremblay

Département de science politique

Université du Québec à Montréal

C.P. 8888, succ. Centre-Ville

Montréal, P.Q. H3C 3P8

Autres membres du Conseil d'administration Guy Lachapelle (Concordia), Manon Trem- blay (Ottawa), Pierre Martin (Montréal), Daniel Latouche (INRS), Antonia Maioni (Ottawa), Guy Bédard (UQAM), Jean Mercier (Laval)

Invité(e)s

Guy Laforest (Laval), Richard Vernon (Western Ontario), codirecteurs de la Revue canadienne de science politique

Réjean Pelletier (Laval), directeur de la Revue québécoise de science politique

Gary O'Brien (Senate of Canada/Sénat du Canada), Délégué de l'Association canadienne de science politique

Elaine Dupré, agente d'administration, La SQSP (UQAM) 


\section{POLITIQUES PROVINCIALES COMPAREES \\ Sous la direction de jean Crete \\ touis M. Imbeau cuy Lachapelle}

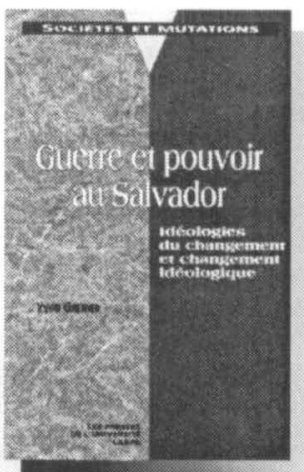

$$
\begin{aligned}
& \text { GTCETre }
\end{aligned}
$$

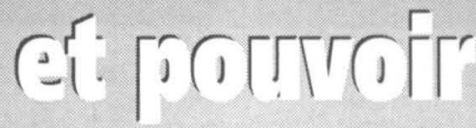

$$
\begin{aligned}
& \text { E. ए厂 }
\end{aligned}
$$

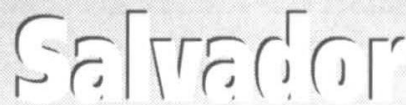

\section{Yvon Grenier}

34 \$, 350 pages

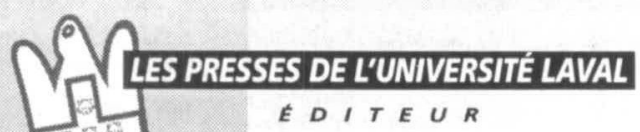

EDITEUR

En vente chez

DISTRIBUTION DE LMAES UNIVERS

845, rue Marie-Victorin, Saint-Nicolas (Québec) G0S 3 LO Tél.: (418) 831-7474 Interurbain: (800) 213-7474 Téléc: (418) 831-4021 